\title{
List of Most Commonly Used Symbols
}

$\bar{A}=$ surface area of a solid or solids per unit volume of pore solution

$a=$ activity

$\hat{C}=$ concentration in terms of mass per unit volume of total sediment (solids plus water)

$\hat{C}_{s}=$ concentration of a solid component in terms of mass per unit volume of total sediment

$C_{s}=$ concentration of a solid component in terms of mass per unit mass of total solids

$\bar{C}=$ concentration of an adsorbed component in terms of mass per unit mass of total solids

$C=$ concentration of a dissolved component in terms of mass per unit volume of pore solution

$C_{e q}=$ saturation equilibrium concentration

$C_{\infty}=$ concentration out in solution; concentration at depth where $\partial C / \partial x \rightarrow 0$ (asymptotic concentration)

$C_{0}=$ concentration at $x=0$

$D=$ generalized diffusion coefficient $;$ molecular diffusion coefficient in water (area per unit time)

$D_{s}=$ molecular diffusion coefficient in sediment including the effects of tortuosity (area of sediment per unit time)

$D_{w c}=$ wave and current mixing (diffusion) coefficient

$D_{B}=$ biodiffusion coefficient for solids

$D_{I}=$ irrigation coefficient

$D_{B I}=$ biodiffusion coefficient for pore water $\left(=D_{B}+D_{I}\right)$

$F=$ formation factor (in molecular diffusion); concentration conversion factor $\left(=\bar{\rho}_{s}(1-\phi) / \phi\right)$

$G=$ concentration of non-diffusable organic matter (expressed as mass of organic carbon per unit mass of total solids) which can be decomposed to $\mathrm{CO}_{2}, \mathrm{H}_{2} \mathrm{O}$, and/or $\mathrm{CH}_{4}$ via a given bacterial process or set of processes; also Gibbs free energy

$J=$ diffusion flux in general (mass per unit area per unit time)

$J_{s}=$ diffusive flux in a sediment (mass per unit area of sediment per unit time)

$K^{\prime}=$ linear adsorption isotherm $\left(K^{\prime}=\bar{C} / C\right)$ 
$K=$ dimensionless linear adsorption isotherm $\left(=F K^{\prime}\right)$; thermodynamic (activity) equilibrium constant

$K_{c}=$ concentration solubility product constant

$k=$ rate constant $\left(\right.$ time $\left.^{-1}\right)$

$L=$ depth (thickness) of the zone of bioturbation

$\mathscr{L}=$ stoichiometric coefficient for sulfate reduction (usually $=1 / 2$ )

$Q=$ water flux in a sediment in terms of volume of water per unit area of sediment per unit time

$R=$ rate of a chemical reaction in mass per unit volume per unit time

$\bar{R}=$ rate of chemical reaction occurring on the surfaces of solids

$\mathscr{R}=$ rate of mass sedimentation (mass per unit area per unit time)

$r=$ spherical or cylindrical coordinate

$r_{c}=$ radius of a spherical crystal, concretion, etc.

$t=$ time

$U=$ generalized water flow velocity (usually lateral) relative to particles

$v=$ velocity of burial of water below the sediment-water interface

$v_{g}=$ vertical water flow velocity relative to particles

$v=$ molar volume

$x=$ depth in sediment below the sediment-water interface (sometimes depth below the base of the zone of bioturbation)

$y=$ lateral dimension in a sediment

$\gamma=$ activity coefficient for a dissolved species

$\lambda=$ radioactive decay constant

$\bar{\rho}_{s}=$ mean density of total sediment solids

$\rho_{w}^{*}=$ mass of $\mathrm{H}_{2} \mathrm{O}$ per unit volume of pore solution

$\sigma=$ specific interfacial (surface) free energy

$\phi=$ porosity (connected porosity) in volume of pore water per unit volume of total sediment (solids plus water); also used for total porosity

$\Omega=$ saturation state of pore solution (equals ion activity product divided by equilibrium ion activity product (solubility product))

$\omega=$ velocity of burial of solid particles below the sediment-water interface

$\left.\begin{array}{r}\phi_{x} \\ v_{x} \\ \omega_{x}\end{array}\right\}=$ asymptotic values of $\phi, v$, and $\omega$ at depth where $\partial \phi / \partial x \rightarrow 0$ 